(where $\theta$ is a function of $a$ and $t$ ) will be

$$
\theta=a \pi^{-1 / 2} \phi .
$$

If, however,

$$
\bar{\theta}(a, p) \equiv \int_{0}^{\infty} e^{-p t} \theta(a, t) d t
$$

equations (3) transform into

$$
\frac{\partial^{2} \bar{\theta}}{\partial a^{2}}=4\left(p+b^{2}\right) \bar{\theta} ; \quad \bar{\theta} \rightarrow \frac{1}{p}, a \rightarrow 0+
$$

which lead at once to

$$
\bar{\theta}=p^{-1} \exp \left[-2 a \sqrt{\left(p+b^{2}\right)}\right] .
$$

The inversion theorem for the Laplace transform then gives

$$
\theta=\frac{1}{2 \pi i} \int_{\gamma-i \infty}^{\gamma+i \infty} \lambda^{-1} \exp \left[\lambda t-2 a \sqrt{\left(\lambda+b^{2}\right)}\right] d \lambda,
$$

along the usual contour.

By a series of obvious and natural steps, ${ }^{3}$ it is easy to show that this is equal to

$$
\begin{aligned}
\frac{e^{2 a b}}{4 \pi i} \int_{\gamma^{\prime}-i \infty}^{\gamma^{\prime}+i \infty} \lambda^{-1} \exp [\lambda t & -2(a+b t) \sqrt{\lambda}] d \lambda+\frac{e^{-2 a b}}{4 \pi i} \int_{\gamma^{\prime \prime}-i \infty}^{\gamma^{\prime \prime}+i \infty} \lambda^{-1} \exp [\lambda t-2(a-b t) \sqrt{\lambda}] d \lambda, \\
= & \frac{e^{2 a b}}{2}\left[1-\operatorname{erf}\left(\frac{a}{\sqrt{ } \hat{t}}+b \sqrt{t}\right)\right]+\frac{e^{-2 a b}}{2}\left[1-\operatorname{erf}\left(\frac{a}{\sqrt{ } \bar{t}}-b \sqrt{ } t\right)\right],
\end{aligned}
$$

and it can be verified that this satisfies (3).

\title{
NOTE ON A FORMULA FOR THE SOLUTION OF AN ARBITRARY ANALYTIC EQUATION*
}

By HERBERT E. SALZER (Mathematical Tables Projec!, Nere York City)

In a recent note $\mathrm{D}$. $\mathrm{R}$. Blaskett and $\mathrm{H}$. Schwerdtfeger ${ }^{-1}$ give a fairly well known expansion for a root $\alpha$ of the equation $f(z)=0$, as a power series in $f\left(z_{0}\right)$, where $z_{0}$ is near $\alpha$, namely,

$$
\alpha=\sum_{\nu=0}^{\infty} \frac{(-1)^{v}\left[f\left(z_{0}\right)\right]^{\nu}}{\nu !}\left[\frac{d^{v} f^{-1}(w)}{d w^{\nu}}\right]_{v=r\left(z_{0}\right)} .
$$

where $w$ denotes $f(z)$.

Of use in connection with (1) is a paper by Van Orstrand, "Reversion of Power Series," Phil. Mag., (6) 19, 366-376 (1910). Van Orstrand's article deals with the re-

3 H. Jeffreys, Operational methods in mathematical physics, Cambridge, 1931, p. 70.

* Received January 26, 1946.

1 This Quarterly 3, 266-268 (1945) 
version of the special type of power series $y=a_{0} x+a_{1} x^{2}+a_{2} x^{3}+\cdots$ to obtain $x$ as an integral power series in $z \equiv y / a_{0}$, whose coefficients are given as polynomials in $b_{i} \equiv-a_{i} / a_{0}$, as far as the term involving $z^{13}$. Now the explicit expansion for (1) in terms of the derivatives of $f(z)$ at $z_{0}$ can be written down immediately, as far as $\nu=13$, from Van Orstrand's expansion on pp. 369-370, merely by

(A) replacing $b_{i}$ in his formula by $-f^{(i+1)}\left(z_{0}\right) /(i+1) ! f^{\prime}\left(z_{0}\right)$,

(B) replacing his $z$ by $-f\left(z_{0}\right) / f^{\prime}\left(z_{0}\right)$, and

(C) adding the constant term $z_{0}$.

The truth of the last statement is obvious from the fact that when (1) is applied at the origin it yields Van Orstrand's expansion and from the uniqueness of Van Orstrand's expansion.

\section{A NOTE ON..THE CORRECTION OF GEIGER MÜLLER COUNTER DATA*}

\section{By H. B. MANN (Ohio State University)}

The correction of Geiger Müller Counter data has been considered in a previous paper by J. D. Kurbatov and the author. ${ }^{1}$ According to the model described there the following result was proved: If the density of radiation is a constant $a$ and if $\tau$ denotes the resolving time, $B(T)$ the number of discharges during the time $T$; then

$$
B(T)=\frac{a T}{1+a \tau}+\eta,
$$

where $\eta$ is given by

$$
\eta=-a \int_{0}^{T} \epsilon(t) d t
$$

and $\epsilon(t)$ satisfies the conditions

$$
\left.\begin{array}{l}
\epsilon(t)=-a \int_{t-\tau}^{t} \epsilon(x) d x \quad \text { for } \quad t \geqq \tau, \\
\epsilon(t)=1-e^{-a t}-\frac{a \tau}{1+a \tau} \quad \text { for } \quad 0 \leqq t \leqq \tau .
\end{array}\right\}
$$

It was further shown that for $a \tau<1$.

$$
|\eta| \leqq \frac{(a \tau)^{2}}{1-(a \tau)^{2}}\left[1-(a \tau)^{s+1}\right],
$$

where $s$ is the largest integer not larger than $T / \tau$. In this paper an upper bound for $|\eta|$ will be derived without the restriction $a \tau<1$. We shall prove the following inequality:

* Received May 29, 1946. (1945).

${ }^{1}$ J. D. Kurbatov and H. B. Mann, A correction for Geiger Mialler counter data, Phys. Rev. 68, 40-43 